\section{CT Angiography is State-of-the-Art First Vascular Imaging for Subarachnoid Hemorrhage}

The American Journal of Neuroradiology shows different attitudes to state-of-the-art imaging with CT angiography (CTA) after subarachnoid hemorrhage. ${ }^{1,2}$ Kallmes et $\mathrm{al}^{1}$ opine that CTA should not be done for subarachnoid hemorrhage, whereas Lubicz et $\mathrm{al}^{2}$ document state-of-the-art CTA imaging of aneurysms.

Kallmes et $\mathrm{al}^{1}$ enshrine selective conventional angiography (DSA), believing that DSA is still needed regardless of whether a cause for hemorrhage is shown on CTA. They imply that CTA is inadequate for needed details in the management of aneurysms. However, Lubicz et $\mathrm{al}^{2}$ and other authors ${ }^{3}$ point to a high level of accuracy of CTA in the detection of aneurysms not only for diagnosis, but also for characterization. They analyze morphologic features of the aneurysm including size, shape, neck orientation, relationship to parent vessels, and relationship to branches. These criteria are needed for management decisions and are shown on CTA despite claims by Kallmes et $\mathrm{al}^{1}$ that these features are obtainable only on DSA.

Kallmes et $\mathrm{al}^{1}$ describe DSA as having "near perfect accuracy and mortality rate of $0.06 \%$." It is unusual to refer to DSA mortality risks because ischemia is most concerning, especially permanent stroke initiated by DSA. Multiple series show low risks in expert angiography practices. ${ }^{4,5}$ DSA complication rates in general clinical practice are not reported, yet it is suspected that permanent risks for stroke with DSA are higher for those authors who are not publishing their stroke rates. Unpublished information known from discussions with angiographic teams includes too many obvious "strokes on the table" during DSA, more than 1 a month. These occurrences have been unheard of for many years by experts.

Recent literature pays little attention to the variance of serious complications from DSA and to safeguards incorporated into basic angiographic technique, including use of experienced scrub assistants and liberal use of heparin in infusion flush, even for patients with hemorrhage. Routine safeguards used by experts are seldom described in detail in publications of endovascular advances. It is as if every angiographer is presumed safe so that there is no discussion, regardless of whether optimal safeguards are used. There are no standardized precautions; yet, every consent for undergoing DSA stresses the risk for stroke.

CTA has no risk for stroke. It is acquired from approximately 5 to 20 seconds of scanning. It uses less contrast than a full "4-vessel" angiogram. DSA has a sterile setup, requires arterial access, and consists of selective catheter manipulations. 3D neuroangiography was an advance in the 1990s because development produced CTA from angiographic C-arm DSA. ${ }^{6}$ DSA with 3D is CTA acquired differently.

CTA from a CT gantry provides angiographic images of all arteries, whereas DSA with 3D gives CTA-type images for each selective, full DSA needing multiple catheterizations for 30 to 60 minutes. Kallmes et $\mathrm{al}^{1}$ point out resolution data, with a difference in spatial resolution between 0.35 and $0.5 \mathrm{~mm}$ for CTA without the risk for stroke and 0.2 and $0.3 \mathrm{~mm}^{1}$ for $3 \mathrm{D}$ conventional angiography with a risk for stroke. Some vessels are more difficult to catheterize, especially for tortuous arches, with increasing stroke risk, increasing difficulty of catheterization, and prolonged procedure time. ${ }^{4,5}$ As neuroradiologists become more and more facile in handling hundreds of CTA images to show and characterize aneurysms, ${ }^{2,3}$ fewer and fewer DSA examinations are being performed. Cases of subarachnoid hemorrhage without a cause shown on quality CTA become candidates for DSA, to search for early ve- nous drainage of a possible dural arteriovenous fistula with pial venous drainage.

Risks of missing tiny aneurysms hidden by other vessels occur as a combination of reader inexperience, failure to invoke enough angles of view, and failure to fill particular vessels with contrast regardless of DSA or CTA. DSA has a greater risk of incomplete filling of aneurysms ${ }^{7}$ because contrast perfuses only 1 vessel at a time, and there could be contrast dilution or layering with slow filling. Both CTA and DSA with 3D need postprocessing, but CTA source images with a CT gantry are fine axial pictures that are diagnostic with high resolution on their own. Source images for DSA with 3D are multiple-angled views of angiographic projections with limited gain to view all directly; their purpose is to create $3 \mathrm{D}$ image volumes. For efficient viewing in addition to source images, CTA is viewed with sections of multiple maximum intensity projections from multiple angles and sometimes rendered images from 3D volumes. DSA with 3D commonly uses rendered images as primary $3 \mathrm{D}$ images. Rendering smoothes vessel edges to produce nice pictures at a cost of losing fine-edge details; this loss is the same for rendering regardless of whether CTA or DSA examination is performed.

Many neuroradiologists are now expert in precise CTA, ${ }^{2,3}$ practicing with goals to duplicate or surpass the positive aspects of DSA. The conclusion by Kallmes et $\mathrm{al}^{1}$ that CTA cannot be done for acute ruptured aneurysms seems applicable only for those who are expert in DSA but not in CTA advances. Perhaps the conclusion of Kallmes et $\mathrm{al}^{1}$ could be revised to read that, "Those who are not expert in CTA and do not work to a goal of CTA 'near perfection' should continue to rely on conventional angiography." Similarly appropriate is that those who are expert in CTA and who no longer perform enough DSA examinations should not do DSA as if it were a near-perfect, low-risk procedure because risks may be far higher than those of the reported stroke rates. DSA examination for pure diagnostic purposes is less frequent with decreasing skills; a comparison today might be to pneumoencephalography, which is no longer done, is not needed, without safe skills.

Yet DSA remains the basis of endovascular treatments, which are now able to be more focused, less time consuming, and with other potential benefits if CTA is done first for hemorrhage. Patients can now come to the interventional suite with the diagnosis, and details of the aneurysm from CTA can be already available ${ }^{2,3}$ from just a few seconds of scanning. When a CTA examination is done first, a full " 4 -vessel” DSA need not be conducted blinded to the ruptured aneurysm. CTA is an important advance in neurovascular imaging and management, including acutely ruptured aneurysms. These conclusions are opinions based on extended experience with DSA for decades and with CTA examination performed with goals to both match and surpass the value of DSA. The published conclusion of Kallmes et $\mathrm{al}^{1}$ that CTA examination should not be performed for ruptured aneurysms is inappropriate today, except perhaps for those who are not expert with CTA advances.

\section{References}

1. Kallmes DF, Layton K, Marx WF, et al. Death by nondiagnosis: why emergent CT angiography should not be done for patients with subarachnoid hemorrhage. AJNR Am J Neuroradiol 2007;28:1837-38

2. Lubicz B, Levrier M, Francois O, et al. Sixty-four-row multisectional CT angiography for detection and evaluation of ruptured intracranial aneurysms: interobserver and intertechnique reproducibility. AJNR Am J Neuroradiol 28:1949-55 
3. Agid R, Lee SK, Willinsky RA, et al. Acute subarachnoid hemorrhage: using 64-slice multidetector CT angiography to "triage" patients' treatment. Neuroradiology 2006;48:787-94

4. Dion JE, Gates PC, Fox AJ, et al. Clinical events following neuroangiography: a prospective study. Stroke 1987;18:997-1004

5. Willinsky RA, Taylor SM, TerBrugge K, et al. Neurologic complications of cerebral angiography: prospective analysis of 2,899 procedures and review of the literature. Radiology 2003;227:522-28

6. Fahrig R, Fox AJ, Lownie $\mathrm{S}$, et al. Use of a C-arm system to generate true three-dimensional computed rotational angiograms: preliminary in vitro and in vivo results. AJNR Am J Neuroradiol 1997;18:1507-14

7. Jou $\mathrm{LD}$, Mohammed $\mathrm{A}$, Lee $\mathrm{DH}$, et al. 3D rotational digital subtraction angiography may underestimate intracranial aneurysms: filling from two basilar aneurysms. AJNR Am J Neuroradiol 2007;28:1690-92

A.J. Fox

S.P. Symons

R.I. Aviv

Sunnybrook Health Sciences Centre

University of Toronto

Department of Medical Imaging

Divison of Neuroradiology

Toronto, Canada

DOl 10.3174/ajnr.A1049 